\title{
Pericarditis relacionada con influenza tipo A
}

\author{
Influenza A-related pericarditis \\ José Gregorio Arellano Aguilar, * Francisco Sánchez Lezama, ${ }^{\ddagger}$ Olga Cristina Chamberlin Varela ${ }^{\S}$
}

Femenino de 45 años con antecedentes heredofamiliares de cáncer renal en madre, padre hipertenso; no vacunada en el último año. Inició padecimiento 10 días antes con tos seca, escalofrío, diaforesis y astenia, agregándose odinofagia. Acudió en dos ocasiones con facultativos, manejada con antimicrobianos (cefalosporinas y penicilina), AINES y antipiréticos. Durante el sexto día se agregó disnea de medianos esfuerzos, palpitaciones y exacerbación de tos por accesos, principalmente nocturnos, se modificó el an-

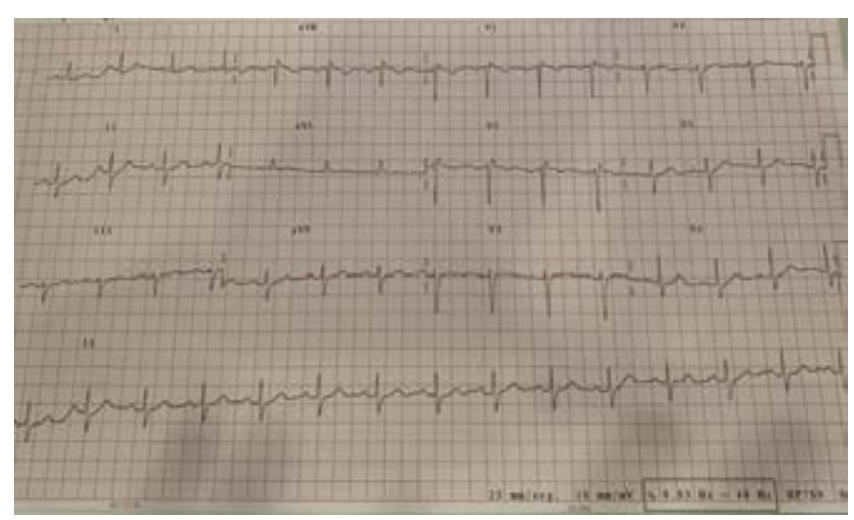

Figura 1: Electrocardiograma en reposo de femenino de 45 años de edad, con influenza tipo A que muestra: ritmo sinusal, frecuencia $90 \mathrm{l} / \mathrm{min}$, eje a $70^{\circ}$; elevación de ST-T cóncavo hacia arriba en múltiples derivaciones (DI, DII, DIII, AVL y AVF, V5 y V6), con mala progresión de R de VI a V4 y S profundas en VI, V2 y V3, correspondiendo a pericarditis.

\footnotetext{
* Especialista en Medicina Interna. División de Medicina del Hospital Ángeles León.

* Cardiólogo. Jefe de Ecocardiografía del Hospital Ángeles León.

$\S$ Radiólogo. Departamento de Imagenología.
}

Hospital Ángeles León. León, Guanajuato, México.

Correspondencia:

Dr. José Gregorio Arellano Aguilar

Correo electrónico: gregareag@gmail.com

Aceptado: 14-06-2019.

www.medigraphic.com/actamedica

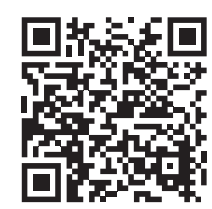

tibiótico sin encontrar mejoría. Acudió a urgencias, donde a la exploración se encontró FC 90 I/min, FR 20 r/min, TA: $100 / 60 \mathrm{mmHg}$, Temp. $38{ }^{\circ} \mathrm{C}$, mucosas orales secas, cuello sin adenomegalias, ingurgitación yugular bilateral, campos pulmonares libres bien ventilados, murmullo vesicular normal, ruidos cardiacos rítmicos de baja intensidad sin fenómenos agregados, abdomen sin visceromegalias, miembros inferiores sin edema, pulsos y llenado capilar normales. Con sospecha clínica de influenza y posible pericarditis se efectuó electrocardiograma, el cual reveló: ritmo sinusal, frecuencia $90 \mathrm{l} / \mathrm{min}$, eje a $70^{\circ}$, elevación de ST-T cóncavo hacia arriba en DI, DII, DIII, AVL y AVF, V5 y $\mathrm{V} 6$ con mala progresión de $\mathrm{R}$ de $\mathrm{VI}$ a $\mathrm{V} 4$ y $\mathrm{S}$ profundas en VI, V2 y V3 (Figura 1). La tele de tórax y pruebas de laboratorio mostraron: cardiomegalia grado I; el laboratorio indicó: citometría hemática, dímero D, troponinas y CPK normales, velocidad de sedimentación globular $28 \mathrm{~mm} /$ hora. La prueba rápida de influenza mostró resultados positivos al antígeno A. Se efectuó tomografía simple de tórax que reveló: datos de pericarditis con engrosamiento del pericardio visceral de $2 \mathrm{~mm}$ y derrame pericárdico calculado en 200 mL (Figura 2). El ecocardiograma confirmó engrosamiento de pericardio visceral y derrame pericárdico sin repercusión hemodinámica con función sistólica conservada (FEVI 70\%) (Figura 3). Fue hospitalizada por seis días, manejada con inhibidor selectivo de neuroaminidasas (oseltamivir 75 mg BID por cinco días), AINE y colchicina a dosis de $0.5 \mathrm{mg} /$ día programada durante tres meses de tolerarse. Fue dada de alta asintomática, con control a 10 días como externa.

La influenza se ha relacionado como factor desencadenante de eventos coronarios agudos, pericarditis y miocarditis, tanto de manera subclínica como con disfunción significativa del ventrículo izquierdo, incluyendo presencia de arritmias y ocasionalmente muerte súbita. ${ }^{1}$ La frecuencia de la afectación cardiaca por influenza es variable $(0-10 \%)$, siendo rara la afectación grave; a la influenza estacional se le atribuye baja toxigenicidad miocárdica. En la pandemia de 2009 se comunicó elevada prevalencia de miocarditis fulminante (62\%) entre los pacientes con miocarditis asociada a H1N1. El ECG es un medio diagnóstico útil, pues son frecuentes las altera- 

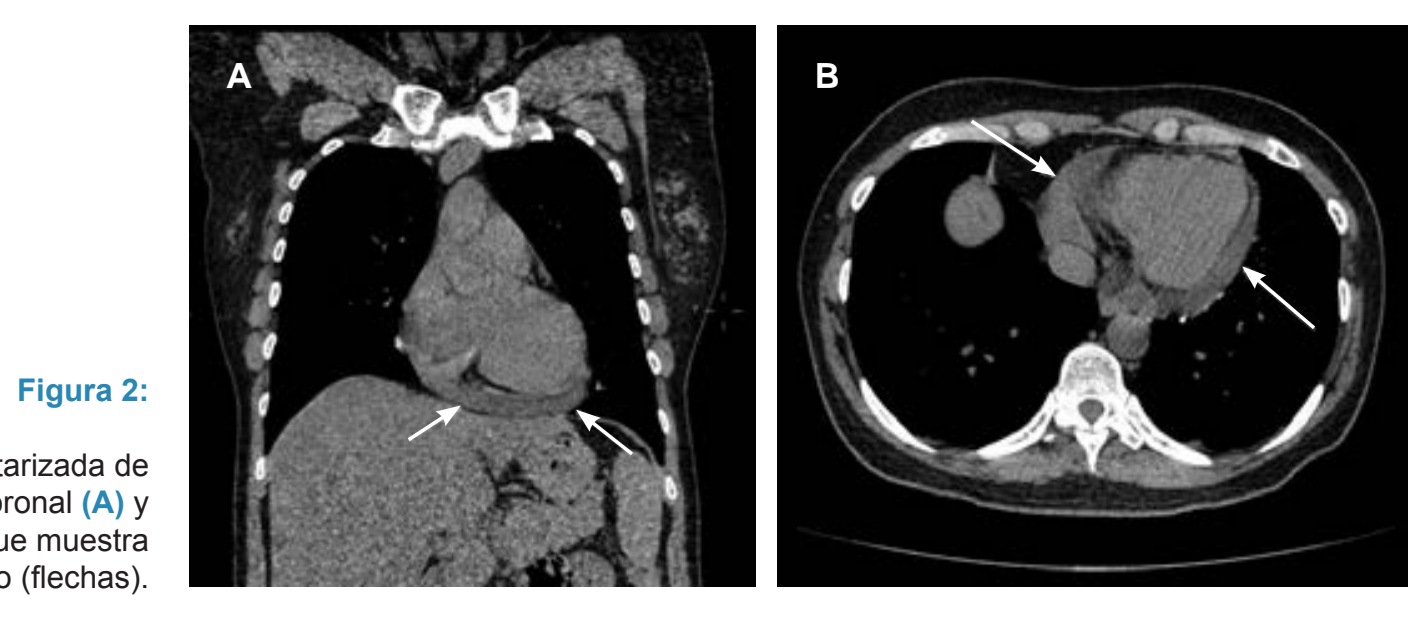

Tomografía computarizada de tórax en cortes coronal (A) y en corte axial (B) que muestra derrame pericárdico (flechas).
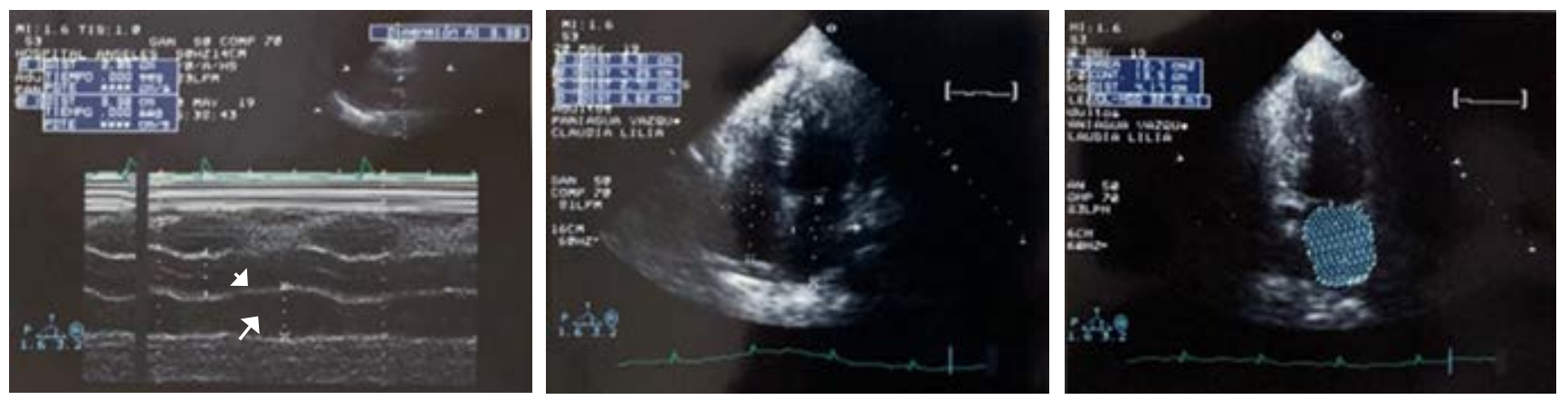

Figura 3: Estudio de ecocardiografía en modo M que revela derrame pericárdico (flecha) y engrosamiento de 2 mm del pericardio visceral (cabeza de flecha), sin repercusión hemodinámica con fracción de expulsión del ventrículo izquierdo de $70 \%$.

ciones de repolarización y los trastornos de conducción. Las alteraciones ecocardiográficas se observan hasta en 90\% de los casos, llegándose a reportar ocasionalmente taponamiento. ${ }^{2}$ El tratamiento consiste en inhibidores de la neuraminidasa. ${ }^{3}$ La biopsia endomiocárdica, aunque sigue siendo la referencia confirmatoria definitiva, no se toma en cuenta actualmente esencial para el diagnóstico y manejo de muchos pacientes. Se considera que el tratamiento precoz puede reducir la mortalidad entre los pacientes hospitalizados por influenza.

\section{REFERENCIAS}

1. Estabragh ZR, Mamas MA. The cardiovascular manifestations of influenza: a systematic review. Int J Cardiol. 2013; 167: 2397-2403.

2. Sidhu SR, Shrama A, Paterson DI, Bainey RK. Influenza H1N1 infection leading to cardiac tamponade in a previously healthy patient: a case report. Res Cardiovasc Med. 2016; 5: e31546.

3. Muthuri SG, Myles PR, Venkatesan S, Leonardi-Bee J et al. Impact of neuraminidase inhibitor treatment on outcomes of public health importance during the 2009-2010 influenza A(H1N1) pandemic: a systematic review and meta-analysis in hospitalized patients. J Infect Dis. 2013; 207: 553-563. 